\title{
Associação entre mortalidade diária por câncer de pulmão e poluição do ar no município do Rio de J aneiro: um estudo ecológico de séries temporais
}

Short term association between lung cancer and air pollution in Rio de J aneiro: a daily time series study

Washington Leite J unger ${ }^{1}$, Antonio Ponce de Leon ${ }^{1}$, Gulnar Azevedo e Silva Mendonça ${ }^{2}$

\section{Resumo}

D iversos estudos têm mostrado associações estatísticas entre níveis de poluição atmosférica e morbidade e/ou mortalidade nas populações. Os principais desfechos de saúde considerados nestes estudos são as doenças respiratórias, cardiovasculares e, mais recentemente, o câncer de pulmão. N este trabal ho investigamos a associação entre poluição do ar e mortalidade por câncer de pulmão em idosos no município do Rio de Janeiro, entre setembro de 2000 a dezembro de 2001. 0 desenho de estudo epidemiológico adotado foi o ecológico com uma abordagem séries temporais diárias. Para ajuste dos fatores de confusão e estimativa do efeito foi utilizada a regressão de Poisson semi-paramétrica. Foram encontrados efeitos estatisticamente significativos para o monóxido de carbono (CO) com riscos relativos $1,130(1,000-1,276)$ e 1,232 $(1,003-1,515)$ para exposição ao CO com defasagem de três dias e acumulada de sete dias respectivamente.

Palavras-chave: N eoplasias pulmonares; Poluição do ar; M ortalidade.

\begin{abstract}
Several studies have presented statistical association between air pollution levels and morbidity and/or mortality in populations. The most common outcomes regarded in these studies are respiratory, cardiovascular diseases and, lately, lung cancer. The aim of this work is to estimate the association between air pollution and lung cancer mortality among elderly people in Rio de Janeiro, from September 2000 to December 2001. An ecological time series approach was used. In order to adjust for confounders and estimate the effects of interest, semiparametric Poisson regression was applied. Statistically significant effects were found for carbon monoxide (CO) but not for the other pollutants analysed. The relative risks were 1.130 (1.000-1.276) and 1.232 (1.003-1.515) for three days lagged and cumulative seven days exposure to $\mathrm{CO}$ respectively.
\end{abstract}

Key words. Lung neoplasms; Air pollution; M ortality.

${ }^{1}$ Instituto de M edicina Social, U niversidade do Estado do Rio de Janeiro

2 Instituto N acional de Câncer - IN CA

Endereço para correspondência: wjunger@ims.uerj.br 


\section{INTRO DUÇÃO}

Estudos recentes utilizando diferentes desenhos epidemiológicos têm fornecido evidências sobre efeitos nocivos da poluição ambiental sobre a saúde da população. Aumentos nos níveis de alguns poluentes atmosféricos têm sido associados com aumento de óbitos e de morbidade por doenças, principalmente, respiratórias e cardiovasculares. ${ }^{1-5} \mathrm{~A}$ preocupação com efeitos deletérios da poluição recebeu maior atenção a partir dos episódios dealta concentração de poluentes ocorridos no ValeM euse, na Bélgica, em 1930, em D onora (Pensilvânia), nos EUA, em 1948, e em Londres, na Inglaterra, em dezembro de 1952. N este último, o número estimado de mortes prematuras foi de 4.000 e se estima que entre dezembro de 1952 emarço de 1953 foram registradas mais de 13.500 mortes acima do esperado para o período. ${ }^{6}$

Com a adoção de medidas visando a reduzir a emissão de poluentes atmosféricos, episódios como estes não foram mais reportados. Atualmente, o aumento nos níveis de poluição é devido principalmente ao crescimento da frota de veículos automotores que hoje, em algumas metrópoles, representa a principal fonte poluidora. 0 surgimento de novas técnicas estatísticas viabilizou 0 estudo dos efeitos da poluição do ar sobre a saúde mesmo quando os níveis de poluição ambiental são menores do que aqueles considerados como nocivos pela O rganização Mundial de Saúde. ${ }^{7}$

Além da consistente relação com o aumento de internações e óbitos por doenças respiratórias e cardiovasculares, alguns estudos epidemiológicos realizados nos últimos 40 anos também têm mostrado a existência de associação entre poluição ambiental e câncer de pulmão. ${ }^{8,9}$ Entretanto, a atenção sobre esta relação é difícil de se precisar muito em decorrência da forte predominância do papel do fumo na etiologia desta doença. ${ }^{10}$

Os principais agentes poluidores ambientais considerados carcinógenos são os subprodutos da queima incompleta dos combustíveis fósseis, sobretudo o diesel. A poluição do ar, principalmente em grandes centros urbanos, é formada por uma mistura bastante variável e complexa destes compostos. Entre eles se destacam 0 benzeno, os benzo[a]pirenos e os compostos orgânicos e inorgânicos. Os hidrocarbonetos policíclicos aromáticos (H AP) incluem vários destes carcinógenos. O s óxidos de nitrogênio podem reagir com os H AP formando potentes substâncias mutagênicas e carcinogênicas. ${ }^{9-11}$

Flutuações de curto prazo nos níveis dos poluentes são determinadas por variações meteorológicas locais e as concentrações destes poluentes normalmente estão correlacionadas no tempo, assim qualquer um dos poluentes pode ser utilizado como um indicador da mistura. Entretanto, em longo prazo, mudançasnaemissão de poluentes podem resultar em modificações substanciais na mistura de poluição do ar. É importante considerar que a presença de agentes carcinógenos na mistura que compõe a poluição atmosférica poderia em parte explicar porque a poluição do ar poderia aumentar o risco de câncer de pulmão e possivelmente de outros cânceres, ${ }^{8,10}$ ou mesmo abreviar a sobrevida de pacientes com estes tipos de câncer. Estudo recente desenvolvido em M ontreal, C anadá, que utilizou metodologia estatística baseada em modelos aditivos generalizados, encontrou efeitos significativos da poluição sobre a mortalidade por câncer de pulmão. ${ }^{12}$

0 objetivo deste trabalho é avaliar o efeito da variação diária nas concentrações de poluentes atmosféricos sobre 0 número diário de óbitos que têm como causa básica câncer de pulmão, na população do município do Rio de Janeiro.

\section{MATERIAL E MÉTO DOS}

\section{DADOS}

A investigação da associação entre a exposição à poluição do ar e o número diário de óbitos por câncer de pulmão, no Rio de Janeiro, foi realizada utilizandose um desenho ecológico de caráter temporal a partir de dados provenientes de fontes secundárias. 0 período de estudo foi de setembro de 2000 a dezembro de 2001.

A partir do Sistema de Informações sobre M ortalidade (SIM), foram geradas séries diárias de óbitos cuja causa básica informada no atestado de óbito foi "neoplasias malignas dos brônquios e dos pulmões" (CID 10, código C 34), para todas as idades e para idosos com 65 anos ou mais.

A estimativa média da exposição diária da população residente no Rio de Janeiro à poluição foi obtida a partir das médias aritméticas das medidas das redes de monitoramento da qualidade do ar da Secretaria M unicipal do M eio Ambiente (SM AC) e da Fundação Estadual de Engenharia de M eio Ambiente (FEEM A). O s poluentes atmosféricos analisados foram: material particulado com até 10 micra de volume aerodinâmico (PM 10), dióxido deenxofre (SO 2) emonóxido de carbono (CO), dióxido de nitrogênio ( $\mathrm{N} O 2$ ) e ozônio (O 3).

Para o ajuste dos dados em relação a fatores meteorológicos foram calculadas as médias aritméticas das medidas de umidade e temperatura (mínima, média e máxima) registradas pelos monitores localizados nos aeroportos civis e militares da cidade e pelo monitor existente no bairro $M$ aracanã. $O s$ dados foram cedidos pelo $D$ epartamento de $M$ eteorologia da Aeronáutica e D epartamento de Climatologia da U niversidade do 
Estado do Rio de Janeiro respectivamente. Dados faltantes foram imputados quando ao menos a metade das observações diárias das concentrações do poluente estavam disponíveis. 0 método de imputação leva em consideração as correlações entre as concentrações de poluentes no espaço e sua estrutura temporal. ${ }^{13}$

\section{ANÁLISE ESTATÍSTICA}

A análise estatística foi realizada utilizando-se modelos de regressão de Poisson semi-paramétrica (modelos aditivos generalizados) implementada no pacote estatístico S-Plus. ${ }^{14} \mathrm{~A}$ estratégia da análise consistiu em modelar a tendência e a sazonalidade da série através de funções splines do tempo; os dias de semana e feriados através de variáveis indicadoras; as condições meteorológicas através de funções splines da temperatura e da umidade relativa do ar. Técnicas de diagnósticos usuais em análise de regressão foram utilizadas para avaliar a inclusão ou exclusão de termos no modelo, bem como a qualidade de ajuste do modelo final. Finalmente, foram adicionados ao modelo os termos correspondentes às concentrações diárias dos poluentes assumindo-se que a associação com a variável resposta é linear. Foram investigadas as associações da exposição no dia corrente, defasagens de até três dias e médias móveis de dois a sete dias anteriores ao desfecho. As médias móveis representam a exposição acumulada nos dias anteriores ao desfecho. D esta forma, foi possível calcular o excesso de óbitos associados às flutuações nos níveis de poluição. ${ }^{15,16}$

Os riscos relativos (RR) para internações correspondem a um aumento de $10 \mu \mathrm{g} / \mathrm{m}^{3}$ nos níveis dos poluentes (exceto para o CO, para o qual se calculou o RR para um aumento de $1 \mathrm{ppm})$. Um nível de significância de $5 \%$ foi adotado nas análises.

\section{RESULTADO}

Estatísticas descritivas das variáveis de desfecho, de poluentes atmosféricos e dos fatores de ajustamento estão dispostas na Tabela 1. Após a imputação não havia observações faltando nas séries de PM 10 e CO e aproximadamente $10 \%$ das observações estavam faltando nas séries de $\mathrm{SO} 2$ e 03 . Entretando, 43\% dos dados de N 02 não estavam disponíveis, tornando as estimativas do efeito deste poluente pouco precisas.

0 gráfico da Figura 1 descreve 0 número diário observado e suavizado de óbitos cuja causa básica é câncer de pulmão. A curva suavizada pela spline não indica tendência de longo prazo. Entretanto, podemos
Tabela 1 . Estatísticas descritivas das variáveis ambientais e desfechos dia a dia

\begin{tabular}{l|c|c|c|c|c|c|c}
\hline & Média & $\mathrm{dp}$ & Min & $\mathrm{P} 10$ & $\mathrm{p} 50$ & $\mathrm{p} 90$ & Max \\
\hline $\mathrm{PM}_{10}$ & 55,85 & 16,75 & 17,94 & 37,41 & 53,52 & 78,13 & 135,23 \\
$\mathrm{SO}_{2}$ & 11,50 & 6,94 & 1,25 & 4,29 & 10,14 & 19,77 & 49,67 \\
$\mathrm{NO}_{2}$ & 51,70 & 24,58 & 14,51 & 25,93 & 48,74 & 79,87 & 207,19 \\
$\mathrm{CO}$ & 1,20 & 0,45 & 0,42 & 0,69 & 1,14 & 1,80 & 3,06 \\
$\mathrm{O}_{3}$ & 29,10 & 16,01 & 2,46 & 9,13 & 31,08 & 48,89 & 85,68 \\
\hline Temperatura máxima & 29,71 & 3,78 & 20,12 & 24,61 & 29,65 & 34,70 & 38,52 \\
Temperatura média & 25,09 & 2,87 & 18,06 & 21,39 & 25,06 & 28,82 & 31,14 \\
Temperatura mínima & 21,67 & 2,74 & 14,90 & 17,98 & 21,62 & 25,06 & 26,86 \\
Umidade Relativa & 77,74 & 6,67 & 60,34 & 68,92 & 77,74 & 86,26 & 96,14 \\
\hline Neoplasias do pulmão & & & & & & & \\
$\quad$ Todas as idades & 3,07 & 1,77 & 0 & 1 & 3 & 5 & 10 \\
$\quad$ 65 anos ou mais & 1,84 & 1,38 & 0 & 0 & 2 & 4 & 8 \\
\hline
\end{tabular}

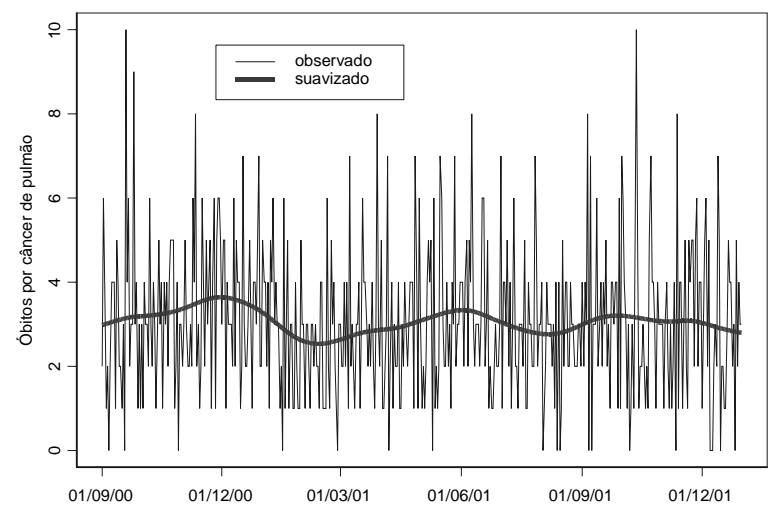

Figura 1- Mortalidade diária por câncer de pulmão durante 0 período de estudo

observar um padrão de sazonalidade com picos nos períodos referentes a extremos de temperatura.

A pós o ajustepelos fatores de confusão (sazonalidade, temperatura e umidade), as variáveis de concentrações diárias dos poluentes foram introduzidas no modelo uma por vez, assim como as defasagens e as médias móveis.

$\mathrm{N}$ a análise da série de óbitos de indivíduos com mais de 65 anos cuja causa básica foi câncer de pulmão, não foi encontrada associação significativa com nenhum dos poluentes analisados. $\mathrm{N}$ o entanto, considerando-se todas as idades, apesar de não terem sido encontradas associações significativas para os poluentes analisados PM 10, SO 2, NO2 e 0 3, a exposição ao monóxido de carbono se mostrou associada ao aumento de óbitos por câncer de pulmão com uma defasagem de três dias ( $R R=1,130$, IC 95\% 1,000; 1,276) e média dos últimos sete dias ( $R R=1,232$, IC 95\% 1,003; 1,515), como mostram a Tabela 2 e a Figura 2. 
Tabela 2 - Riscos relativos e intervalos de confiança para exposição ao $\mathrm{CO}$

\begin{tabular}{l|c|c}
\hline Medida da exposição & RR & IC $(95 \%)$ \\
\hline dia corrente & 1,018 & $(0,898 ; 1,154)$ \\
defasagem de 1 dia & 1,052 & $(0,931 ; 1,189)$ \\
defasagem de 2 dias & 1,084 & $(0,959 ; 1,226)$ \\
defasagem de 3 dias & 1,130 & $(1,000 ; 1,276)$ \\
\hline acumulado 2 dias & 1,051 & $(0,911 ; 1,213)$ \\
acumulado 3 dias & 1,098 & $(0,936 ; 1,288)$ \\
acumulado 4 dias & 1,168 & $(0,982 ; 1,388)$ \\
acumulado 5 dias & 1,176 & $(0,977 ; 1,414)$ \\
acumulado 6 dias & 1,201 & $(0,987 ; 1,462)$ \\
acumulado 7 dias & 1,232 & $(1,003 ; 1,515)$ \\
\hline
\end{tabular}

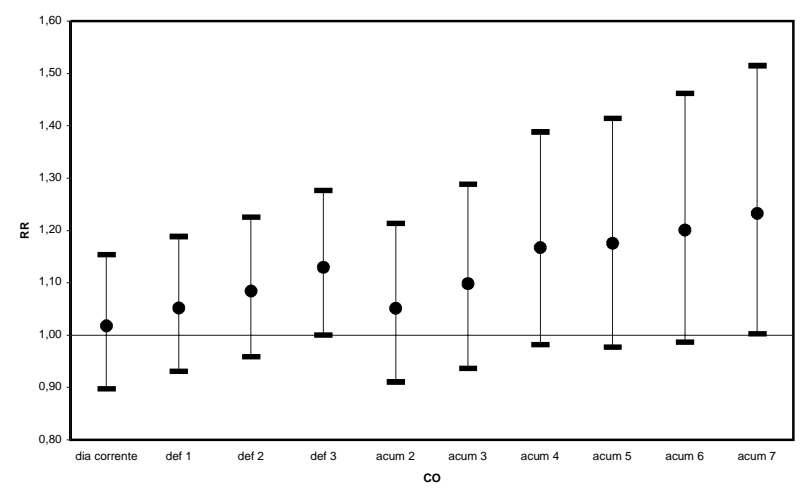

Figura 2 - Riscos relativos e intervalos de confiança para exposição ao CO para defasagens e acumulados

\section{DISCUSSÃO}

A pesar das limitações inerentes ao desenho de estudo utilizado neste trabalho, foi possível encontrar associação estatisticamente significativa entre poluição do ar e mortalidade por câncer de pulmão no município do Rio de Janeiro. Para interpretarmos os resultados obtidos neste estudo devemos considerar que o câncer de pulmão é uma doença crônica e que a informação tendo como causa básica de óbito de pulmão, não permite que se conheça exatamente qual foi 0 evento que levou 0 indivíduo à morte.

Com as restrições impostas pelo desenho epidemiológico utilizado neste estudo, que avalia a relação entre poluição e óbito num período de até sete dias entre exposição e desfecho, não podemos avaliar a poluição do ar como fator envolvido na causalidade da doença uma vez que o processo de carcinogênese é iniciado com grande defasagem de tempo. D e fato, este estudo pode apenas sugerir causalidade entre poluição do ar e complicações que podem ter agravado o quadro clínico levando ao óbito dos pacientes já acometidos pelo câncer de pulmão.

A capacidade do organismo de suportar estresse e se manter estável é maior no indivíduo adulto e diminui com a idade ou doenças. Os efeitos da poluição do ar na saúde envolvem principalmente idosos e portadores de doenças crônicas. ${ }^{17}$ Indivíduos expostos a altas concentrações de monóxido de carbono no ambiente sofrem um aumento da concentração de carboxihemoglobina e diminuição da concentração de oxigênio no sangue. Portadores de doenças pulmonares crônicas como câncer de pulmão e doença pulmonar obstrutiva crônica (D PO C) têm sua capacidade pulmonar diminuída pela doença e não são capazes de manter 0 equilíbrio da pressão de 02 e $\mathrm{CO}$ no sangue. ${ }^{18}$ Este padrão de efeito do CO sobre a mortalidade é coerente com a hipótese de harvesting ou colheita. ${ }^{19}$

A fim de estimar a curva dose-resposta, foi ajustado um modelo para cada poluente e defasagem de exposição e, portanto, um total de 50 testes de hipótese foram realizados. Embora o número de resultados estatisticamente significativos seja próximo do esperado ao nível de $5 \%$, os resultados encontrados neste trabalho são compatíveis com outro estudo dos mesmos autores (ainda não publicado) que investigou a associação entre poluição do ar e mortalidade por DPOC, encontrando riscos relativos em torno de 1,24 e estatisticamente significativos para a exposição média de seis e sete dias anteriores ao desfecho. É possível que o aumento de óbitos em função de aumento de poluição por monóxido de carbono possam ser explicados por mecanismos de restrição pulmonar. $M$ ais estudos que investiguem de forma sistemática e por períodos prolongados de tempo esta relação devem ser desenvolvidos e outros fatores envolvidos na etiologia da doença, e.g. tabagismo, devem ser considerados.

\section{REFERÊNCIAS}

1. Daumas RP, M endonca GA, Ponce AD L. Air pollution and mortality in theelderly in Rio de Janeiro: a time-series analysis. C ad Saúde Pública. 2004;20(1):311-9.

2. GouveiaN, FletcherT. Respiratory diseases in children and outdoor air pollution in Sao Paulo, Brazil: a time series analysis. O ccup Environ M ed. 2000;57(7):477-83.

3. Atkinson RW, Anderson H R, Sunyer J, Ayres J, Baccini M , VonkJM, et al. Acute effects of particulateair pollution on respiratory admissions: resultsfrom APH EA 2 project. Air Pollution and $\mathrm{H}$ ealth: a European Approach. Am J Respir Crit C are M ed. 2001;164(10 Pt 1):1860-6.

4. SametJM , D ominici F, Zeger SL, SchwartzJ, D ockery D W. Thenational morbidity, mortality, and air pollution study. 
Part I: methods and methodologic issues. Res Rep H ealth Eff Inst. 2000;(94 Pt 1):5-14.

5. Ponce D L, Anderson $H R$, Bland JM, Strachan D P, Bower $J$. Effects of air pollution on daily hospital admissionsfor respiratory diseasein London between 1987-88 and 199192. J Epidemiol Community H ealth. 1996;50 Suppl 1:S63-70.

6. Bell M L, D avisD L, Fletcher T. A retrospectiveassessment of mortality from the London smog episode of 1952: the role of influenza and pollution. Environ $\mathrm{H}$ ealth Perspect. 2004;112(1):6-8.

7. Brunekree $B, H$ olgateST. Air pollution and health. Lancet. 2002;360(9341):1233-42.

8. Katsouyanni K, Pershagen $\mathrm{G}$. Ambient air pollution exposure and cancer. Cancer Causes Control. 1997;8(3):284-91.

9. Pershagen G. Air pollution and cancer. IARC Sci Publ. 1990;(104):240-51.

10. Cohen AJ, PopeCA, Speizer FE. Ambient air pollution as a risk factor for lung cancer. Salud Publica M ex. 1997;39(4):346-55.

11. Speizer FE. 0 verview of the risk of respiratory cancer from airborne contaminants. Environ $\mathrm{H}$ ealth Perspect. 1986;70:9-15.

12. Goldberg M S, BurnettRT, Bailar JC 3rd, Brook J, Bonvalot Y, Tamblyn R, et al. Theassociation between daily mortality and ambient air particlepollution in M ontreal, Q uebec. 2.
Cause-specific mortality. Environ Res. 2001;86(1):26-36.

13. J unger WL. Imputação de dados faltando em séries temporaismultivariadas via al goritmo EM . Rio dej aneiro (RJ): Instituto deM atemática eEstatística daU niversidade do Estado do Rio de Janeiro; 2002.

14. $\mathrm{H}$ astie T, Tibshirani R. Generalized additive models. London: Chapman and $\mathrm{H}$ all; 1990.

15. Schwartz J, Spix C, Touloumi G, Bacharova L, Barumamdzadeh T, LeTertreA, et al. M ethodological issues in studies of air pollution and daily counts of deaths or hospital admissions. J Epidemiol Community $\mathrm{H}$ ealth. 1996;50 Suppl 1:S3-11.

16. Katsouyanni K, SchwartzJ, Spix C, Touloumi G, Zmirou $D, Z$ anobetti $A$, et al. Short term effects of air pollution on health: aEuropean approach using epidemiologic timeseries data: the APHEA protocol. J Epidemiol Community H ealth. 1996;50 Suppl 1:S12-8.

17. Frank $R$, Tankersley $C$. Air pollution and daily mortality: a hypothesis concerning the role of impaired homeostasis. Environ H ealth Perspect. 2002;110(1):61-5.

18. C asarett LJ, D oull J, Klaassen CD , Watkins JB. C asarett and D oull's toxicology: the basic science of poisons: companion handbook. N ew York: M cGraw-H ill; 1999.

19. Schwartz J. Isthereharvesting in the association of airborne particles with daily deaths and hospital admissions? Epidemiology. 2001;12(1):55-61. 